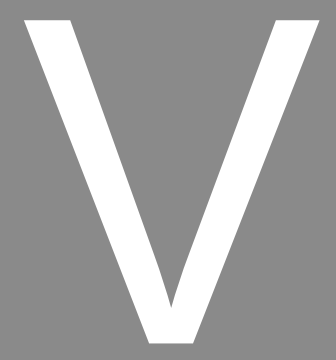

\title{
PERCEPCIÓN DE LOS ESTUDIANTES SECUNDARIOS ACERCA DE LAS CLASES DE BIBLIA
}

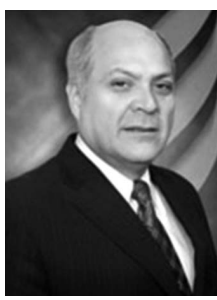

\section{Vicente León}

Licenciado en Educación por la Universidad de Antofagasta, Chile. Magíster en Administración Educacional por la Universidad de Antofagasta. Doctor en Educación por la Universidad de Montemorelos. Ponente internacional. Actualmente se desempeña como director de Posgrado de la Universidad Adventista de Chile, donde también realiza actividades de investigación, produciendo publicaciones y ponencias en diversas revistas de nivel internacional y con carácter científico. 


\section{Resumen}

Este estudio tuvo como objetivo caracterizar las percepciones que los estudiantes, de primero a cuarto medio de los colegios secundarios adventistas de la Unión Chilena, tienen acerca del desarrollo de las clases de Biblia. También se buscó establecer si había diferencias de percepción según género y según la religión de los participantes. La investigación se realizó durante el año 2012 en los 24 colegios secundarios que la Iglesia adventista sostiene en Chile. El total de participantes fue de 4.941. El instrumento empleado para el desarrollo del estudio fue creado ex profeso y sometido a las pruebas de validez de contenido y de constructo mediante análisis factorial que arrojó tres factores llamados "Interés en la clase de Biblia", "Metodología empleada en la clase de Biblia" y "Evaluación que se hace en la clase de Biblia". Los indicadores del instrumento fueron evaluados en la escala: nunca, rara vez, a veces, generalmente y siempre. Los resultados mostraron que, de los 29 indicadores que componen el instrumento, solo 8 de ellos se acercan en promedio a la categoría "generalmente" y 21 señalan "a veces". La prueba t de student mostró que existen diferencias de género en la dimensión "Interés en la clase de Biblia" y en la dimensión cómo perciben la "Evaluación" que se hace en ella. El ANOVA de un factor mostró diferencias de percepción en los estudiantes según su origen religioso en las dimensiones "Interés por la clase de Biblia", en la "Metodología que se emplea en ella" y en la "Evaluación que se hace en dicha clase". Como conclusión general se puede indicar que la clase de Biblia es una tarea pendiente que necesita ser rediseñada y mejorada notablemente porque el estudiantado en general no la está percibiendo a un nivel óptimo.

Palabras clave: Enseñanza, Biblia, metodología y evaluación.

\section{Abstract}

This study aimed to characterize the perceptions of students from first to fourth through Adventist secondary schools Chilean Union have on the development of Bible classes. It also sought to establish whether there were differences in perception by gender and according to the religion of the participants. The research was conducted in 2012 in 24 high schools argues that the Adventist Church in Chile. The total number of participants was 4,941. The instrument used for the development of the study was created expressly and subjected to testing content validity and construct using factor analysis yielded three factors called "Interest in Bible class", "Methodology for Bible class" and "Evaluation is done in Bible class." The indicators of the instrument were evaluated on a scale: never, rarely, sometimes, often and always. The results showed that, of the 29 indicators that make up the instrument, only 8 of which are on average closer to the category "generally" and 21 said "sometimes". The Student $t$ test showed that there are gender differences in the dimension "Interest in Bible class" and how they perceive the dimension "Assessment" made it ". The way ANOVA showed differences of perception in students according to their religious origin in the dimensions "Interest in Bible class" in the "Methodology that is used in it" and the "Evaluation is done in this class" . As a general conclusion may indicate that Bible class is a pending task that needs to be redesigned and greatly improved because the students in general not being perceived at an optimal level.

Key words: Teaching, Bible, methodology and evaluation. 


\section{La enseñanza de la Biblia}

El Decreto Supremo de Educación 924 (1983) del Ministerio de Educación de Chile establece la obligatoriedad de la enseñanza de religión en todos los establecimientos educacionales del país. El Artículo 5 señala que "los establecimientos particulares confesionales, ofrecerán a sus alumnos la enseñanza de la religión a cuyo credo pertenecen y por cuya razón han sido elegidos por los padres de familia al matricular a sus hijos". Sin duda que desde la perspectiva legal los colegios adventistas han sido beneficiados con esta normativa que los faculta para impartir religión, en cuya clase obviamente es prioritario el estudio de la Biblia. Al respecto White (1971), señala que:

La Biblia debería ser el primer libro de texto del niño. De este libro, los padres han de dar sabias instrucciones. La palabra de Dios ha de constituir la regla de la vida. De ella los niños han de aprender que Dios es su Padre; y de las hermosas lecciones de su Palabra han de adquirir un conocimiento de su carácter. Por la inculcación de sus principios, deben aprender a hacer justicia y juicio. (p. 105)

La pregunta que se puede hacer es: ¿por qué hay que estudiar la Biblia en la clase de religión? White (1989), establece que "el propósito del auténtico estudio de la Biblia es conocer personalmente a Cristo y tener un conocimiento experimental de la salvación como se revela en la Biblia". Para hacer posible la confrontación divino-humana, de tal manera que Dios pueda hablar a través de su Palabra, las buenas nuevas de salvación a los estudiantes. De esta manera llegarán a entender que el amante Dios, Creador y Redentor, ha actuado en favor de la raza perdida, y provee poder sobrenatural para traer al hombre de nuevo a una relación restaurada consigo mismo. Esta es una transacción personal intensa de confesión y aceptación por parte del alumno.

Las clases de Biblia deberían desafiar a los estudiantes a dedicar sus talentos y energías a Dios, con el propósito de hacer una contribución importante en el mundo (King, 2006).

La Biblia debe ser usada como texto de estudio en las escuelas del sistema educativo adventista dado que "la Biblia hará para la mente y para la moral lo que no pueden hacer los libros de ciencia y filosofía. Como libro destinado a disciplinar y fortalecer el intelecto, ennoblecer, purificar y refinar el carácter, es sin rival”. (White, 1971, p. 408)

Gillespie (2006), declara que el 63\% de los estudiantes de los grados 6 al 12 en las escuelas adventistas dicen que el profesor de Biblia es un factor 
importante en sus decisiones de fe. White (1971), agrega que la Biblia "usada como libro de texto en nuestras escuelas, resultará mucho más eficaz que cualquier otro libro para guiar sabiamente en los asuntos de esta vida, así como para ayudar al alma a ascender por la escalera que alcanza el cielo". (p. 434)

White (1988), recalca que

La Biblia es el gran libro de texto de Dios, su gran educador. El fundamento de toda ciencia verdadera se halla en la Biblia. Cada rama del conocimiento puede ser hallada escudriñando la Palabra de Dios. Y sobre toda otra cosa contiene la ciencia de todas las ciencias, la ciencia de la salvación. La Biblia es la mina de las inescrutables riquezas de Cristo. (p. 80)

El estudio de la Biblia en nuestras escuelas dará a los estudiantes ventajas especiales. Los que recibían en su corazón los santos principios de la verdad obrarán con energía creciente. Ninguna circunstancia podrá alterar su resolución de alcanzar la norma más elevada que sea posible. Y lo que hayan recibido lo impartirán a otros. Mientras beben de la fuente de agua viva, brotarán de ellos raudales vivos para bendecir y refrigerar a otros. (White, 1971, p. 436)

Newell (2003), señala que uno puede elegir leer la Biblia exclusivamente como un libro sobre religión, o puede creer que Dios se revela a sí mismo en su palabra para cada uno. Es evidente que el profesor de Biblia puede y debe hacer la diferencia para que los estudiantes se encuentren con Dios a través del estudio de su Palabra.

\section{Metodología}

Este estudio fue empírico, cuantitativo y descriptivo. Buscó caracterizar la percepción que los estudiantes secundarios tienen acerca del desarrollo de las clases de Biblia en sus colegios y se compararon las dimensiones que componen el instrumento de medición respecto del género y de la religión que profesan los estudiantes.

\section{Participantes}

La población estuvo conformada por todos los estudiantes de secundaria $\left(1^{\circ}\right.$ a $\left.4^{\circ}\right)$ matriculados en los colegios secundarios adventistas en el año 2012, de los diferentes territorios administrativos que componen la Unión 
Chilena, que estuvieron presente en el día en que se aplicó la encuesta. Este estudio fue de tipo censal; en la Tabla 1 se puede apreciar la participación del estudiantado en la investigación, que fue en total de 4941.

Tabla 1. Participantes en el estudio según colegio al que pertenecen

\begin{tabular}{lr}
\hline Colegio & Participantes \\
\hline Adventista Buenaventura & 124 \\
Adventista Concepción & 253 \\
Adventista de Angol & 103 \\
Adventista de Antofagasta & 130 \\
Adventista de Arica & 218 \\
Adventista de Calama & 229 \\
Adventista de Chillán & 605 \\
Adventista de Copiapó & 269 \\
Adventista de lquique & 213 \\
Adventista de Molina & 167 \\
Adventista de Pitrufquén & 99 \\
Adventista de Punta Arenas & 111 \\
Adventista de Quilpué & 207 \\
Adventista de Talcahuano & 296 \\
Adventista de Temuco & 220 \\
Adventista La Cisterna & 136 \\
Adventista La Serena & 112 \\
Adventista Las Condes & 80 \\
Adventista Los Ángeles & 720 \\
Adventista Porvenir & 155 \\
Adventista Santiago Norte & 157 \\
Adventista Santiago Poniente & 84 \\
Adventista Santiago Sur & 189 \\
Adventista Talca & 4941 \\
\hline Total &
\end{tabular}

\section{Instrumento}

La recolección de los datos se llevó a cabo mediante el instrumento Percepción de los Estudiantes Secundarios sobre la Clase de Biblia (PESCB). Dicho instrumento fue creado ex profeso para este estudio en el año 2011. El proceso de creación se desarrolló tomando en cuenta los principios filosóficos caracterizadores de la educación adventista, cuyo eje central es precisamente la clase de Biblia. En principio las declaraciones que lo componían fueron revisados por varios expertos hasta llegar a una propuesta de 50 ítems, luego del cual fue sometido a la validación de jueces que evaluaron la claridad y pertinencia del 
mismo, quedando finalmente en 33 ítems. A continuación se realizó una prueba piloto para medir la validez y confiabilidad. El proceso anterior permitió eliminar cuatro indicadores cuyas cargas factoriales fueron menores que 5, quedando finalmente en 29 indicadores. La aplicación de la prueba piloto del instrumento condujo a un análisis de validez de constructo para lo cual se corrió un análisis factorial ( $\mathrm{KMO}=, 981$, Barttlet $=85709,653, \mathrm{gl}=406, \mathrm{p}=, 000)$ resultando en tres factores ortogonales (rotación Varimax con Normalización Kaiser que resultó en cinco iteraciones) que explican el 63,07\% de la varianza total. 16 indicadores de la dimensión "Interés por las clases de Biblia" tienen cargas factoriales entre ,513 y ,784 mientras que los siete indicadores de la dimensión "Metodología empleada en la clase de Biblia" tienen cargas factoriales entre ,511 y ,782. Los indicadores de la tercera dimensión, "Evaluación de la clase de Biblia", quedó con seis indicadores con cargas factoriales entre ,598 y,733. La Tabla 2 presenta los tres factores con sus respectivos indicadores y cargas factoriales.

Respecto a la confiabilidad de las tres dimensiones se obtuvo para la escala "Interés por la clase de Biblia" un coeficiente alpha de Cronbach igual a ,956, para la escala "Metodología empleada en la clase de Biblia" ,884 y en la escala de "Evaluación de las clases de Biblia" el alpha fue de ,881. El coeficiente de confiabilidad conjunta fue de, 965 . Cada indicador fue contestado en una escala en donde 1 es nunca, 2 rara vez, 3 a veces, 4 generalmente y 5 siempre.

Tabla 2. Factores extraídos en la rotación varimax.

\begin{tabular}{|c|c|c|c|}
\hline Declaraciones & Factor 1 & Factor 2 & Factor 3 \\
\hline $\begin{array}{l}\text { Las clases de Biblia me animan a participar en actividades de } \\
\text { servicio al prójimo. }\end{array}$ & ,660 & & \\
\hline $\begin{array}{l}\text { Las clases me motivan para aprender cada vez más sobre la } \\
\text { Biblia. }\end{array}$ & 720 & & \\
\hline $\begin{array}{l}\text { Las clases de Biblia me motivan porque estoy conociendo más } \\
\text { de Dios y su plan para mi vida. }\end{array}$ & ,782 & & \\
\hline $\begin{array}{l}\text { Me interesa asistir a las clases de Biblia porque me resulta } \\
\text { atractiva, amena y dinámica. }\end{array}$ & ,622 & & \\
\hline $\begin{array}{l}\text { Los contenidos tratados en las clases de Biblia son apropiados } \\
\text { para mis necesidades espirituales. }\end{array}$ & ,699 & & \\
\hline $\begin{array}{l}\text { En las clases de Biblia se aprecia entusiasmo por participar y } \\
\text { opinar sobre los contenidos que se tratan (en ella). }\end{array}$ & ,513 & & \\
\hline $\begin{array}{l}\text { Los contenidos de las clases de Biblia están relacionados con } \\
\text { mi preparación para la vida. }\end{array}$ & 707 & & \\
\hline $\begin{array}{l}\text { Creo que las clases de Biblia le agregan calidad a la educación } \\
\text { secundaria (media) que recibo en mi colegio. }\end{array}$ & ,656 & & \\
\hline
\end{tabular}




\section{Declaraciones}

Factor 1 Factor 2 Factor 3

Los contenidos de las clases de Biblia me ayudan a comprender

, 725

la necesidad de tener un espíritu de servicio y de abnegación hacia otra personas.

Los contenidos de las clases de Biblia me incentivan a tomar decisiones bajo la ética cristiana.

Los contenidos de las clases de Biblia me ayudan a entender la voluntad de Dios para mi vida.

Los contenidos de las clases de Biblia me ayudan a descubrir mis intereses y necesidades espirituales.

En las clases de Biblia percibo que la salvación es lo más importante en mi vida.

Los contenidos de las clases de Biblia fomentan mi desarrollo espiritual como persona y como hijo de Dios.

Las clases de Biblia me han ayudado a tener un verdadero acercamiento a Jesús.

Las clases de Biblia me han ayudado a aprender a estudiar la Biblia en forma personal.

La metodología utilizada en las clases de Biblia son activas y participativas.

Las actividades desarrolladas en las clases de Biblia son variadas.

En las clases de Biblia se usan diferentes estrategias de ense-

ñanza.

Los trabajos desarrollados en las clases de Biblia son dirigidos y supervisados.

Los temas desarrollados en las clases de Biblia me dan la oportunidad de discutirlos con mis compañeros.

Los recursos didácticos utilizados en las clases de Biblia son variados.

En las clases de Biblia se usan ilustraciones para comprender los contenidos.

Las evaluaciones que se aplican en las clases de Biblia miden

mis conocimientos, habilidades y actitudes.

En las clases de Biblia se usan diferentes tipos de procedimientos evaluativos (pruebas, trabajos grupales, investigaciones, disertaciones etc.).

Recibo retroalimentación en mis evaluaciones de las clases de Biblia.

Los criterios de evaluación de las clases de Biblia son adecuados a los objetivos de la materia.

Las evaluaciones en las clases de Biblia apuntan a mi desarrollo de actitudes positivas.

Los contenidos evaluados se corresponden con los desarrollados en las clases de Biblia.

,762

,782

, 772

, 517

,702

,511 


\section{Resultados}

A continuación se presentan los resultados de este estudio. El total de encuestados fue de 4.941 de los cuales el $47,4 \%$ son varones (2.292) y el $52,6 \%$ mujeres (2.539). El 42,2\% son adventistas (1765), el 27,6\% son evangélicos (1154), el 22,9\% son católicos (958) y el 7,2\% señala no profesar religión (301). El promedio de edad de los participantes fue de 16 años.

En la Tabla 3 se puede apreciar que en el factor "Interés por la clase de Biblia" la declaración que presenta la media más baja $(M=2,95)$ corresponde a "me interesa asistir a las clases de Biblia porque me resulta atractiva, amena y dinámica" y la declaración que alcanzó el promedio más alto $(3,87)$ fue "en las clases de Biblia percibo que la salvación es lo más importante en mi vida". Cada una de las 16 declaraciones de esta dimensión fue evaluada, a lo menos, con un 1 (nunca) y, a su vez, todas recibieron alguna calificación 5 (siempre). Considerando la escala utilizada para contestar a cada una de las preguntas, se puede decir que hay 10 declaraciones que se ubican en "a veces" y solo seis declaraciones están próximas a "generalmente". El promedio de los 16 indicadores de esta dimensión fue de 3,43.

Tabla 3. Dimensión interés por la clase de Biblia

\begin{tabular}{|c|c|c|c|c|c|}
\hline Declaraciones & $\mathrm{N}$ & Mín. & Máx. & Media & D.E. \\
\hline $\begin{array}{l}\text { Me interesa asistir a las clases de Biblia } \\
\text { porque me resulta atractiva, amena y } \\
\text { dinámica. }\end{array}$ & 4899 & 1 & 5 & 2,95 & 1,280 \\
\hline $\begin{array}{l}\text { Las clases de Biblia me animan a partici- } \\
\text { par en actividades de servicio al prójimo. }\end{array}$ & 4917 & 1 & 5 & 3,04 & 1,184 \\
\hline $\begin{array}{l}\text { En las clases de Biblia se aprecia entu- } \\
\text { siasmo por participar y opinar sobre los } \\
\text { contenidos que se tratan (en ella). }\end{array}$ & 4905 & 1 & 5 & 3,05 & 1,232 \\
\hline $\begin{array}{l}\text { Las clases de Biblia me han ayudado a } \\
\text { aprender a estudiar la Biblia en forma } \\
\text { personal. }\end{array}$ & 4854 & 1 & 5 & 3,13 & 1,300 \\
\hline $\begin{array}{l}\text { Las clases me motivan para aprender cada } \\
\text { vez más sobre la Biblia. }\end{array}$ & 4934 & 1 & 5 & 3,30 & 1,230 \\
\hline $\begin{array}{l}\text { Los contenidos de las clases de Biblia me } \\
\text { incentivan a tomar decisiones bajo la ética } \\
\text { cristiana. }\end{array}$ & 4902 & 1 & 5 & 3,45 & 1,231 \\
\hline $\begin{array}{l}\text { Las clases de Biblia me han ayudado a } \\
\text { tener un verdadero acercamiento a Jesús. }\end{array}$ & 4889 & 1 & 5 & 3,46 & 1,240 \\
\hline $\begin{array}{l}\text { Los contenidos tratados en las clases de } \\
\text { Biblia son apropiados para mis necesida- } \\
\text { des espirituales. }\end{array}$ & 4866 & 1 & 5 & 3,46 & 1,239 \\
\hline
\end{tabular}




\begin{tabular}{|c|c|c|c|c|c|}
\hline Declaraciones & $\mathrm{N}$ & Mín. & Máx. & Media & D.E. \\
\hline $\begin{array}{l}\text { Los contenidos de las clases de Biblia me } \\
\text { ayudan a descubrir mis intereses y necesi- } \\
\text { dades espirituales. }\end{array}$ & 4897 & 1 & 5 & 3,46 & 1,229 \\
\hline $\begin{array}{l}\text { Creo que las clases de Biblia le agregan } \\
\text { calidad a la educación secundaria (media) } \\
\text { que recibo en mi colegio }\end{array}$ & 4918 & 1 & 5 & 3,49 & 1,288 \\
\hline $\begin{array}{l}\text { Los contenidos de las clases de Biblia me } \\
\text { ayudan a comprender la necesidad de } \\
\text { tener un espíritu de servicio y de abnega- } \\
\text { ción hacia otra personas. }\end{array}$ & 4897 & 1 & 5 & 3,51 & 1,185 \\
\hline $\begin{array}{l}\text { Los contenidos de las clases de Biblia } \\
\text { están relacionados con mi preparación } \\
\text { para la vida. }\end{array}$ & 4897 & 1 & 5 & 3,59 & 1,192 \\
\hline $\begin{array}{l}\text { Los contenidos de las clases de Biblia } \\
\text { fomentan mi desarrollo espiritual como } \\
\text { persona y como hijo de Dios. }\end{array}$ & 4900 & 1 & 5 & 3,65 & 1,216 \\
\hline $\begin{array}{l}\text { Las clases de Biblia me motivan porque } \\
\text { estoy conociendo más de Dios y su plan } \\
\text { para mi vida. }\end{array}$ & 4920 & 1 & 5 & 3,67 & 1,244 \\
\hline $\begin{array}{l}\text { Los contenidos de las clases de Biblia me } \\
\text { ayudan a entender la voluntad de Dios } \\
\text { para mi vida. }\end{array}$ & 4905 & 1 & 5 & 3,85 & 1,210 \\
\hline $\begin{array}{l}\text { En las clases de Biblia percibo que la sal- } \\
\text { vación es lo más importante en mi vida }\end{array}$ & 4894 & 1 & 5 & 3,87 & 1,259 \\
\hline
\end{tabular}

En la dimensión sobre la "Metodología empleada en la clase de Biblia", la declaración "los temas desarrollados en las clases de Biblia me dan la oportunidad de discutirlos con mis compañeros" es la que obtuvo el promedio más bajo $(M=3,06)$ y la declaración "los trabajos desarrollados en las clases de Biblia son dirigidos y supervisados" obtuvo la media más alta $(3,41)$. Según la escala utilizada para contestar, las siete declaraciones que componen este factor marcan "a veces". En esta dimensión las siete declaraciones también fueron evaluadas desde la categoría "nunca" (1) hasta la categoría "siempre" (5). El promedio de los siete indicadores de esta dimensión es de 3,14. 


\section{Vicente León}

Tabla 4. Dimensión metodología empleada en la clase de Biblia

\begin{tabular}{|c|c|c|c|c|c|}
\hline Declaraciones & $\mathbf{N}$ & Mín. & Máx. & Media & D.E. \\
\hline $\begin{array}{l}\text { Los temas desarrollados en las clases de Biblia me dan } \\
\text { la oportunidad de discutirlos con mis compañeros. }\end{array}$ & 4903 & 1 & 5 & 3,06 & 1,289 \\
\hline $\begin{array}{l}\text { La metodología utilizada en las clases de Biblia son } \\
\text { activas y participativas. }\end{array}$ & 4887 & 1 & 5 & 3,08 & 1,254 \\
\hline $\begin{array}{l}\text { Los recursos didácticos utilizados en las clases de Biblia } \\
\text { son variados. }\end{array}$ & 4855 & 1 & 5 & 3,09 & 1,228 \\
\hline $\begin{array}{l}\text { En las clases de Biblia se usan diferentes estrategias de } \\
\text { enseñanza. }\end{array}$ & 4895 & 1 & 5 & 3,10 & 1,258 \\
\hline $\begin{array}{l}\text { Las actividades desarrolladas en las clases de Biblia son } \\
\text { variadas. }\end{array}$ & 4895 & 1 & 5 & 3,11 & 1,237 \\
\hline $\begin{array}{l}\text { En las clases de Biblia se usan ilustraciones para com- } \\
\text { prender los contenidos. }\end{array}$ & 4895 & 1 & 5 & 3,18 & 1,254 \\
\hline $\begin{array}{l}\text { Los trabajos desarrollados en las clases de Biblia son } \\
\text { dirigidos y supervisados. }\end{array}$ & 4874 & 1 & 5 & 3,41 & 1,272 \\
\hline
\end{tabular}

En la Tabla 5 se puede apreciar que en el factor "Evaluación de la clase de Biblia" la declaración con la media más baja $(M=3,05)$ corresponde a "recibo retroalimentación en mis evaluaciones de las clases de Biblia" y la declaración con el promedio más alto $(3,63)$ fue "Los contenidos evaluados se corresponden con los desarrollados en las clases de Biblia". Las seis declaraciones fueron evaluadas utilizando desde la categoría "nunca" (1) hasta la categoría "siempre" (5). Considerando la escala utilizada para contestar a cada una de las preguntas se puede decir que hay 4 declaraciones que se ubican en "a veces" y solo dos declaraciones están próximas a "generalmente". El promedio de los seis indicadores de esta dimensión es de 3,34.

Tabla 5. Dimensión evaluación de la clase de Biblia

\begin{tabular}{lrrrrr}
\hline Declaraciones & N & Mín. & Máx. & Media & D.E. \\
\hline $\begin{array}{l}\text { Recibo retroalimentación en mis evaluaciones de } \\
\text { las clases de Biblia. }\end{array}$ & 4882 & 1 & 5 & 3,05 & 1,241 \\
$\begin{array}{l}\text { En las clases de Biblia se usan diferentes tipos de } \\
\text { procedimientos evaluativos (pruebas, trabajos } \\
\text { grupales, investigaciones, disertaciones etc.). }\end{array}$ & 4915 & 1 & 5 & 3,21 & 1,347 \\
$\begin{array}{l}\text { Las evaluaciones que se aplican en las clases de } \\
\text { Biblia miden mis conocimientos, habilidades y }\end{array}$ & 4909 & 1 & 5 & 3,25 & 1,272 \\
$\begin{array}{l}\text { actitudes. } \\
\begin{array}{l}\text { Los criterios de evaluación de las clases de Biblia } \\
\text { son adecuados a los objetivos de la materia. }\end{array}\end{array}$ & 4896 & 1 & 5 & 3,40 & 1,210 \\
$\begin{array}{l}\text { Las evaluaciones en las clases de Biblia apuntan a } \\
\text { mi desarrollo de actitudes positivas }\end{array}$ & 4917 & 1 & 5 & 3,54 & 1,240 \\
$\begin{array}{l}\text { Los contenidos evaluados se corresponden con los } \\
\text { desarrollados en las clases de Biblia. }\end{array}$ & 4911 & 1 & 5 & 3,63 & 1,273 \\
\hline
\end{tabular}


Para establecer si existe diferencia de género en cada una de las dimensiones, se procedió a sumar los 16 indicadores del factor "Interés de los estudiantes en la clase de Biblia"; lo mismo fue realizado con las otras dos dimensiones (metodología y evaluación de las clases de Biblia) con siete y seis indicadores respectivamente. Se utilizó la prueba t de student para muestras independientes y se encontró que en el factor "Interés en la clase de Biblia" existe una diferencia significativa entre hombres y mujeres ( $t(4829)=-5,494, p$ $=, 000)$. Los hombres ( $M=53,32$ ) obtuvieron una media inferior a las mujeres ( $M$ $=55,73)$. En el factor de "Metodología de la clase de Biblia" no se encontraron diferencias de género. En la dimensión "Evaluación de la clase de Biblia" los resultados mostraron que existe una diferencia significativa $(\mathrm{t}(4795)=-3,129$, $p=, 002)$. Las damas obtuvieron una media más alta $(M=20,25)$ que la de los varones $(M=19,71)$.

Respecto de la religión que dicen profesar los estudiantes, el ANOVA de un factor mostró que existen diferencias significativas en el factor "Interés en la clase de Biblia" $\left(F_{(3,4174)}=188,826\right.$ sig. $\left.=.000\right)$, en la dimensión "Metodología de la clase de Biblia" $\left(F_{(3,4172)}=45,130\right.$ sig. $\left.=.000\right)$ y en el factor "Evaluación de las clases de Biblia" $\left(F_{(3,4170)}=88,447\right.$ sig. $\left.=.000\right)$.

La prueba post hoc de Games-Howell permitió observar que en el factor "Interés en las clases de Biblia" los estudiantes adventistas $(M=58,91)$ difieren significativamente $(p=, 000)$ de los evangélicos $(M=55,76)$, de los católicos $(M=53,27, p=, 000)$ y de los que no profesan alguna religión ( $M=$ $38,53, p=, 000)$. En el factor "Metodología de la clase de Biblia" los estudiantes adventistas $(M=22,62)$ difieren significativamente $(p=, 014)$ con los católicos $(M=21,84)$ y con los estudiantes que no profesan ninguna religión ( $M=17,90$ $\mathrm{p}=, 000)$. En el factor "Evaluación de las clases de Biblia", los adventistas ( $M=$ $21,19)$ difieren significativamente $(p=, 000)$ de los evangélicos $(M=20,31)$, de los católicos $(M=19,54, p=, 000)$ y de los que no profesan religión $(M=15,46$, $p=, 000)$.

\section{Conclusiones y recomendaciones}

En virtud de los resultados obtenidos se puede concluir que las clases de Biblia necesitan ser revisadas en cada una de las dimensiones que estableció este estudio. Los indicadores de cada uno de los tres factores no lograron ser evaluados en la categoría "siempre", más bien quedaron en la categoría "a veces". Se podría señalar que lo anterior se genera por tener grupos de alumnos que difieren notablemente en sus creencias y en el tipo de fe que profesan. No 
obstante, tanto los estudiantes adventistas como los evangélicos, católicos y los que señalan no profesar alguna religión evaluaron los distintos indicadores utilizando los cinco calificaciones que componen la escala del instrumento. Esto significa que las percepciones de los estudiantes son muy diversas no importando la religión a la que pertenecen.

Las damas mostraron más "Interés en la clase de Biblia" que los varones, y también perciben que la "Evaluación que se hace en ella" es acorde con las características de la materia. El aspecto que destaca en este estudio es que los estudiantes adventistas se perciben más interesados en la clase de Biblia, perciben positivamente la metodología empleada para el desarrollo de las clases y la evaluación es acorde con los objetivos de la misma. Esto podría ser considerado como muy bueno. Sin embargo, no hay que olvidar que solo el $42,2 \%$ son adventistas y el $57,8 \%$ no lo es. Esto podría significar que la clase de Biblia no está alcanzando adecuadamente sus objetivos. Sería muy interesante que la clase de Biblia llegue a ser tan apreciada por todos los estudiantes, independientemente de su religión.

Al considerar los promedios de cada dimensión, se puede señalar que la dimensión "Metodológica" es la que tiene el promedio más bajo. Esta situación puede deberse al hecho de que quienes hacen clases de Biblia, por lo general, son los capellanes, quienes han recibido una preparación teológica y no pedagógica. Esta situación necesita ser subsanada, a objeto de que se empleen mejores métodos o estrategias que atiendan las particularidades de esta materia tan fundamental dentro del currículum escolar adventista.

Según los resultados obtenidos se puede apreciar que la clase de Biblia no despierta, por sí sola, el interés de los estudiantes. Es necesario realizar un trabajo delicado y a conciencia para despertar el aprecio por el desarrollo de esta asignatura. Si los estudiantes lograran comprender lo que está en juego, conocer a Dios Creador, Sustentador y Redentor, la actitud y disposición, sin duda, serían otras. Sin embargo, el desafío principal está en cómo se desarrolla la docencia de estas clases, lo que implica mejorar la formación de los que imparten dicha clase, proveyéndoles de materiales didácticos adecuados para impartir su enseñanza. Como indica White (1989).

El resultado de tal estudio de la Biblia será mentes bien equilibradas; porque se desarrollarán armoniosamente las facultades físicas, mentales y morales. No habrá parálisis en el conocimiento espiritual. Se aguzará la comprensión, se despertará la sensibilidad, se sensibilizará la conciencia: se purificarán las simpatías y los sentimientos, se creará una atmósfera moral y mejor, y se impartirá un nuevo poder para resistir la tentación. (p. 99) 
En muchas asignaturas la meta es dominar la materia, pero en el caso de las clases de Biblia los alumnos deben ser dominados por la materia. Esto quiere decir que el estudio de materias de teología debe ser mucho más que simplemente un tema a analizar. Debe ser integral para la vida. Hay un peligro muy real de que en las presentaciones de los preceptos cristianos en clases los "fríos hechos" reemplacen a las "palabras de vida". Otro peligro, relacionado con el anterior, es que el profesor se entusiasme tanto con las intrincadas cuestiones teológicas que pierda contacto con las realidades de la adolescencia. Muchos profesores de Biblia creen ver una magnífica oportunidad de sumergirse en asuntos teológicos que carecen de relevancia para las mentes juveniles.

La clase de Biblia no es cualquier clase; por lo tanto, es urgente adentrarse en el desarrollo de esta y hacer los cambios que esta materia del currículum escolar necesita. Para el sistema educativo adventista la clase de Biblia es fundamental y cobra mucho sentido desde el punto de vista de lo que se pretende alcanzar a través de ella. Por esta razón, Gillespie (2006), recalca que se necesitan "profesionales entrenados, con el don de la enseñanza y un conocimiento apropiado de la escritura y del pensamiento teológico son la mejor opción para enseñar a los estudiantes".

Se debe investigar qué intereses está despertando la clase de Biblia, qué metodología se está utilizando para el desarrollo de la clase y qué tipo de evaluación se hace en ella.

No hay temas más importantes presentados en el currículo adventista que los de la clase de Religión. Sin embargo, probablemente no hay temas más en peligro de perderse en la apatía a causa de una constante repetición de lo obvio. Muchos alumnos llegan pensando que ya saben todo sobre la Biblia, cuando en verdad ignoran profundamente sus problemas reales. La clase de Biblia es un desafío importante que tiene el sistema educativo adventista, bien vale la pena prestar la atención que le corresponde, es una responsabilidad ante Dios, los estudiantes, los padres y la Iglesia.

Vicente León Universidad Adventista de Chile email: vicenteleon@unach.cl 


\section{Referencias}

Gillespie, V. (2006). El ministerio de la enseñanza de la Biblia. Revista de Educación Adventista. 23, 21-24.

King, G. (2006). ¿Deberían los colegios y universidades adventistas exigir la clases de religión? Revista de Educación Adventista, 23, 16-20.

Newell, R. (2003). Teaching the Bible along the devotional/academic faultline: An incarnational approach to the quarrel between love and knowledge. Teaching Theology and Religion, 4, 190-197.

Ministerio de educación. (1983). Decreto Supremo 924. Santiago.

White, E. G. (1971). Consejos para los maestros, padres y alumnos acerca de la educación cristiana. Buenos Aires: ACES.

White, E. G. (1988). Exaltad a Jesús. Miami: APIA.

White, E. (1989). Mente, carácter y personalidad. (Vol. 1). México: APIA. 